\title{
State of the Art : Transforaminal Approach for Percutaneous Endoscopic Lumbar Discectomy under Local Anesthesia
}

\author{
Koichi Sairyo', Hiroshi Egawa', Tetsuya Matsuura', Mitsuhiko Takahashi', \\ Kosaku Higashino', Toshinori Sakai', Naoto Suzue', Daisuke Hamada', \\ Tomohiro Goto', Yoichiro Takata', Toshihiko Nishisho', Yuichiro Goda', \\ Ryosuke Sato', Takahiko Tsutsui', Ichiro Tonogai', Kenji Kondo', Fumitake Tezuka', \\ Kazuaki Mineta', Kosuke Sugiura', Makoto Takeuchi', and Akira Dezawa ${ }^{2}$ \\ ${ }^{1}$ Department of Orthopedics, the University of Tokushima, Tokushima, Japan, ${ }^{2}$ Department of Ortho- \\ pedic Surgery, Teikyo University Mizonokuchi Hospital, Kawasaki, Japan
}

\begin{abstract}
Minimally invasive percutaneous endoscopic discectomy (PED) with a transforaminal approach under local anesthesia was started in the late 20th century. As the procedure requires a skin incision of only $8 \mathrm{~mm}$, it is the least invasive disc surgery procedure at present, and owing to advances in instruments and optics, the use of this technique has gradually spread. In Japan, Dr. Dezawa from Teikyo University Mizonokuchi Hospital introduced this technique in 2003. Thanks to his efforts, the number of surgeons who can perform PED has increased, although the number of active PED surgeons is still only around 20. The first author (K.S.) started PED in 2010. In this review article, we explain the state-of-the-art PED transforaminal technique for minimally invasive disc surgery and present three successful cases. J. Med. Invest. 61 : 217-225, August, 2014
\end{abstract}

Keywords : Herniated nucleous pulposus, lumbar spine, surgery, percutaneous endoscopic discectomy, local anesthesia

\section{INTRODUCTION AND HISTORY}

Figure 1 shows a typical magnetic resonance imaging (MRI) of a herniated nucleus pulposus (HNP) of the lumbar spine. The conventional surgical approach to HNP is open removal, which includes laminotomy, flavectomy, and HNP fragment removal, in a procedure known as Love's technique. In the late 20th century, surgeons started to attempt the procedure in a minimally invasive manner under

Received for publication February 27, 2014 ; accepted March 18, 2014.

Address correspondence and reprint requests to Koichi Sairyo, $\mathrm{MD}, \mathrm{PhD}$, Professor and Chairman, Department of Orthopedics, the University of Tokushima, 3-18-15 Kuramoto, Tokushima 770-8503, Japan and Fax : +81-88-633-0178. a microscope and with a special retractor (1). After 1990, the technique was further developed by utilizing a spinal endoscope and a tubular retractor. This endoscope-assisted trans-tubular surgery was eventually established by Foley et al. (2) and Destandeau (3) in what is now known as microendoscopic discectomy (MED). With these procedures, surgeons can access the HNP through the interlaminar space, that is, via the interlaminar approach. Figure 1 shows the site of this approach.

Another means of accessing the intracanal HNP is the transforaminal approach, also shown in Figure 1. The HNP is accessed through the intervertebral foramen (Figure 2). Hijikata (4) started to utilize this approach in what became the conventional technique called percutaneous discectomy. The benefit 


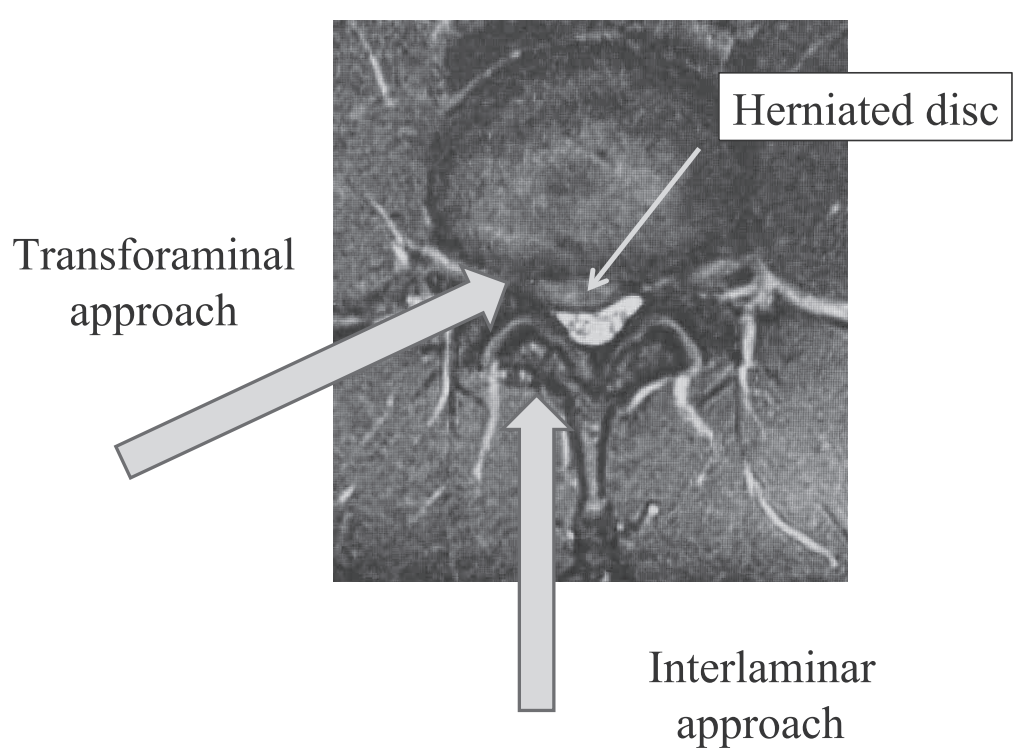

Figure 1 : Magnetic resonance imaging (MRI) of a herniated nucleous pulposus, and the location of two approaches.

of the approach is that it is conducted under local anesthesia with minimal invasiveness. Kambin was another pioneer of this approach (5). However, without an endoscope, it was difficult to remove an HNP located inside the canal. Percutaneous endoscopic discectomy (PED) developed out of these techniques.

From the later 1980 s, a trial was started by Kambin, Schreiber, and Leu to utilize an endoscope or arthroscope in the transforaminal approach (6, 7). Thanks to their efforts and to the development of spinal instruments for endoscopic surgery, percutaneous endoscopic lumbar discectomy was established and has since spread worldwide. The establishment of the current single-portal endoscopic discectomy was made possible by the efforts of Yeung et al. (8-10). The use of a spinal endoscope improves reliability when removing an HNP compared with Hijikata's conventional technique. Recently, Dezawa and colleagues (11-14) further established an advanced technique using a high-speed drill which enables most HNPs to be removed using a minimally invasive PED system.

In regard to transforaminal surgery in Japan, the technique actually originated with the work of Dr. Hijikata (4), but utilizing the endoscope in his technique was advanced in a number of other countries (6-10). Dr. Dezawa from Teikyo University Mizonokuchi Hospital, Japan adopted this technique in 2003, and thanks to his efforts, the number of PED surgeons in Japan has gradually increased, although as of 2013, the number of active PED surgeons is still only around 20 or so. The first author (K.S.) started using this technique under Dr. Dezawa's guidance in 2010. In this review article, we explain the state-of-the-art PED transforaminal technique under local anesthesia and present three successful cases.

\section{SURGICAL TECHNIQUE}

Surgery is conducted with the patient in the prone position on a standard spine frame. The cannula insertion point is determined before surgery on computed tomography and MRI. The optimum location is 8 to $12 \mathrm{~cm}$ from the midline, although this varies among individuals ; for thin patients, around $8 \mathrm{~cm}$ from the midline is appropriate. While viewing the $\mathrm{C}$-arm image intensifier, local anesthesia is performed with $1 \%$ lidocaine. For safe access to the HNP through the so-called safety triangle (Figure 2 ), the walking technique is effective. To avoid injuring the nerve root, which exits the cranial side of the intervertebral foramen (Figure 2), the puncture needle must first make contact with the caudal pedicle. Then, using the walking technique, the needle can be safely inserted into the intervertebral disc. This maneuver is called "walking technique", because the needle walks from the pedicle to the disc. This procedure requires $10-15 \mathrm{ml}$ lidocaine : the most important point is to limit the amount of lidocaine near the exiting nerve root. Exposing the exiting nerve root to lidocaine can injure the nerve. Figure 3 indicates the location of the skin incision and the operative scene in transforaminal 


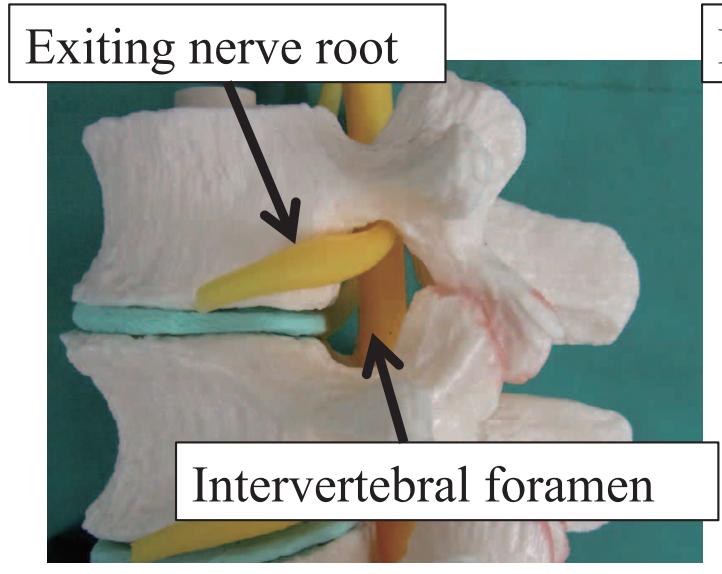

Lateral view

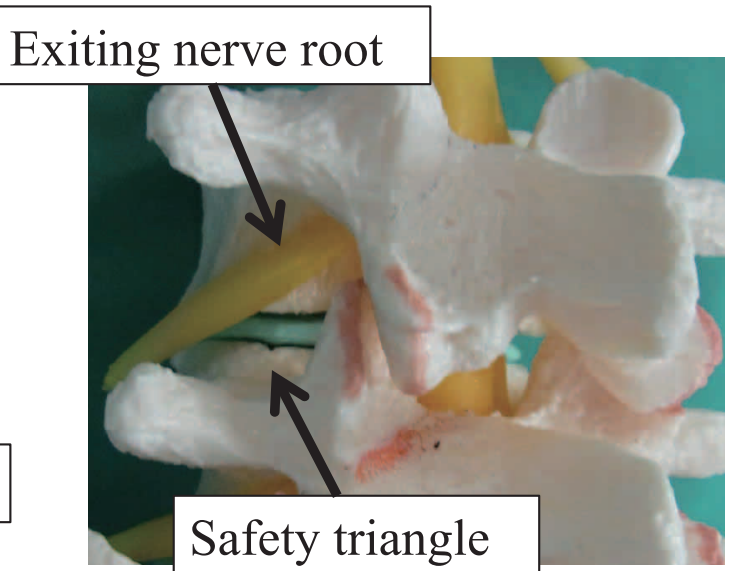

Oblique view

Figure 2 : Anatomical location of the exiting nerve root, intervertebral foramen, and safety triangle.

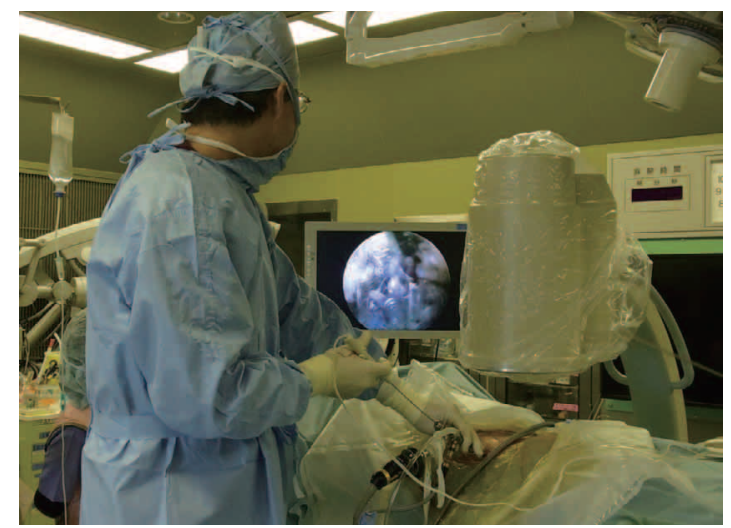

PED procedure

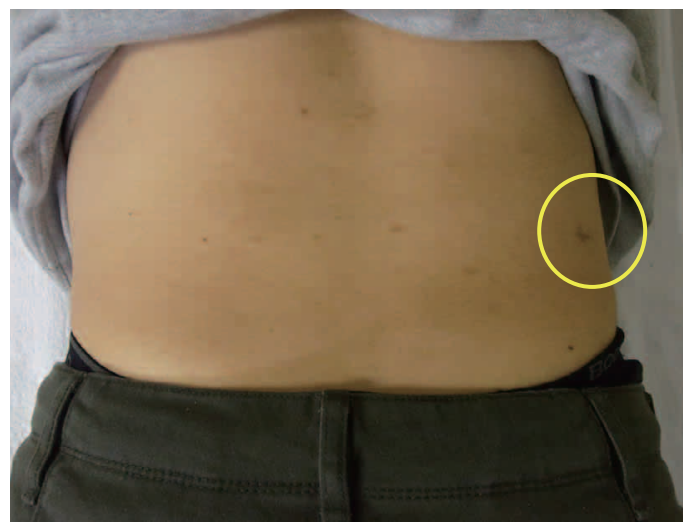

Surgical scar

Figure 3 : Operative scene in percutaneous endoscopic discectomy (PED) and the surgical incisional scar.

\section{PED surgery.}

Next, discography is conducted with indigo carmine to dye the nucleus pulposus and herniated mass blue (Figure 4). The annulus fibrosus stays white and the epidural space red due to the presence of vessels. These differences in color clearly differentiate the herniated mass, allowing it to be safely removed. A guide pin is inserted into the disc through the puncture needle, and the obturator and cannula are inserted sequentially through the $8-\mathrm{mm}$ skin incision. After inserting the cannula, the disc fragment at the base of the herniated mass is removed. Then, according to the inside-out and handdown technique (Figure 5), the cannula is moved toward the epidural space, the herniated mass is removed (Figure 6), and pulsation of the dural tube is confirmed as the indicator of the decompression.

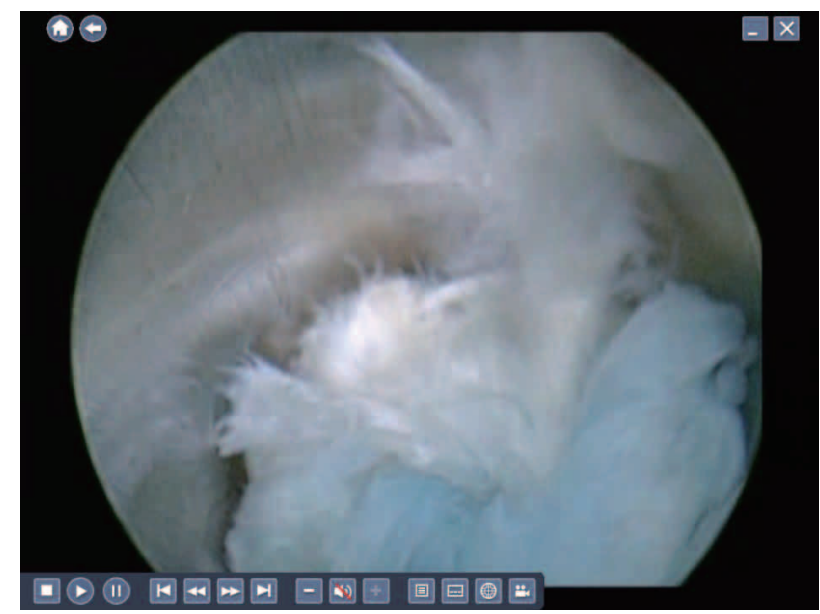

\section{Endoscopic View}

Figure 4 : Endoscopic view of the disc. The nucleous pulposus is dyed blue. 


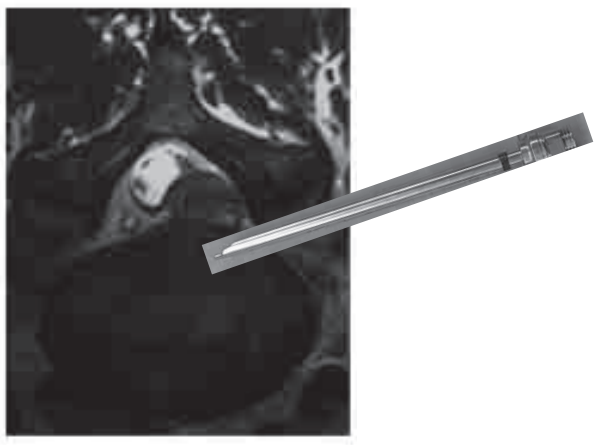

Starting location of a cannula

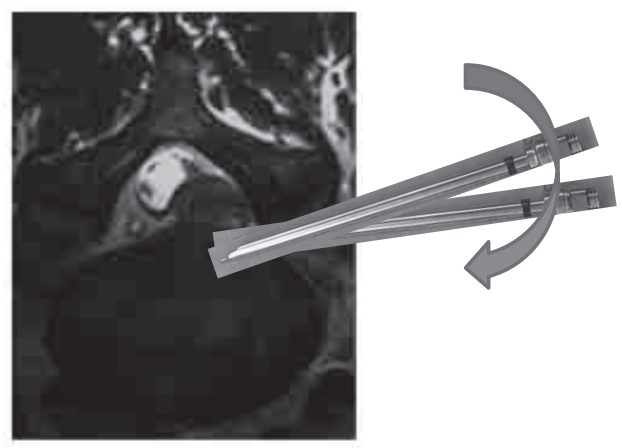

Hand-down technique

Figure 5 : Inside-out technique of the transforaminal approach of PED.

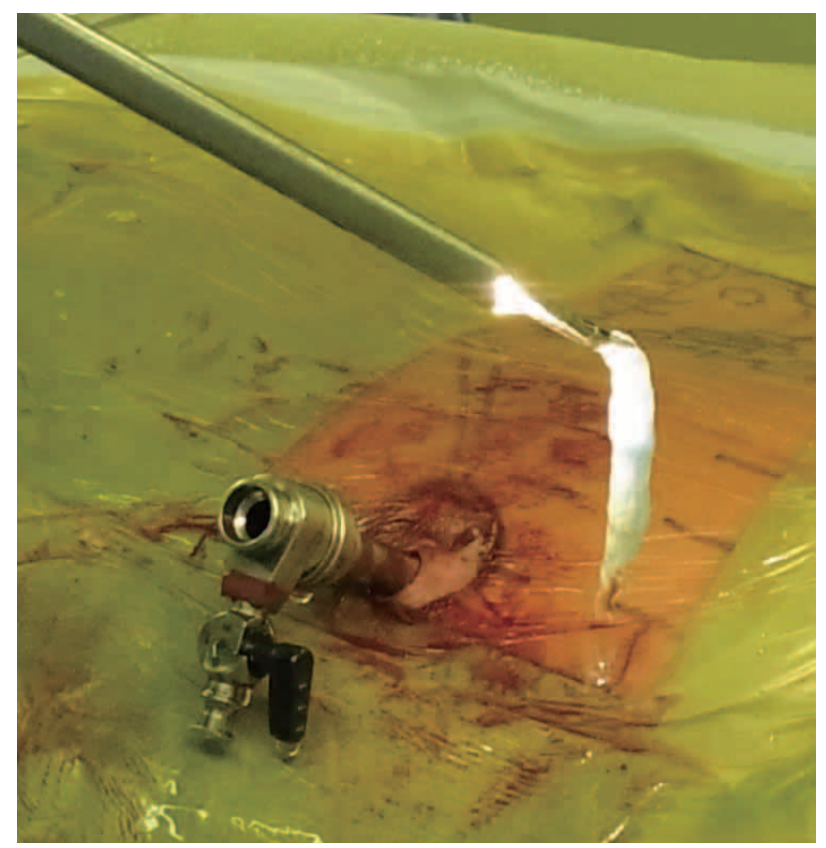

\section{Removal of a HNP mass through a cannula}

Figure 6 : Removal of the herniated nucleous pulposus.

\section{POST-SURGICAL SCHEDULE}

The patient can start standing and walking 1-2 $\mathrm{h}$ after surgery. Sometimes during local anesthesia, however, lidocaine infiltrates the exiting nerve and the patient experiences numbness, in which case bed rest is needed. Patients are typically discharged the 1 to 3 days after the surgery. Desk work is allowed 4-5 days after surgery, while no heavy labor is permitted for $6-8$ weeks to prevent recurrence.
In most cases, sports activity is allowed 5-6 weeks after the surgery. However, returning to contact sports should be carefully determined.

\section{SURGICAL INDICATION}

\section{1 : Intracanal HNP at the L1/2 to L4/5 level without migration}

This HNP is a good indication for the transforaminal approach. Migrated HNP and HNP at the L5/S1 level are also possible indications ; however, an additional technique is required to remove them.

\section{2 : Migrated HNP}

Migrated HNPs are not complete contraindications. However, pediculotomy, which is a technically demanding procedure, is required to remove an upward- or downward-migrated HNP (15). For HNPs that migrate even further into the hidden zone, Dezawa et al. proposed the translaminar approach with PED (12).

\section{3 : Intracanal HNP at the L5/S1 level with a high iliac crest}

Among the 307 cases reported by Yeung and Tsou (9), over 50\% had an HNP at the L5/S1 level. In so-called "high iliac" cases, the iliac crest can hinder level insertion of the cannula through the intervertebral foramen. Therefore, the standard technique cannot be applied and the cannula cannot be inserted into the canal at the appropriate position. To address this issue, Lee et al. (16) proposed the foraminoplastic approach, which enables the cannula to be inserted at the appropriate position after enlarging the intervertebral foramen by foraminoplasty. 
Moreover, to remove the HNP from this location, small incised MED using PED system should be selected (11).

\section{4: Far-lateral HNP}

For an HNP located outside the canal, the cannula can be inserted directly into the HNP, making it comparatively easy to remove the mass since there is no need to insert the cannula through the intervertebral foramen. In fact, a far-lateral HNP can be removed at all lumbar levels by the posterolateral approach under local anesthesia $(17,18)$.

\section{$5:$ Recurrent HNP}

Safely removing a recurrent $\mathrm{HNP}$ at the same level by Love's or micro-Love's technique or MED is technically demanding due to adhesions and scar tissue surrounding the nerve root and HNP. For PED, the transforaminal approach can be performed as the initial operation, which is the strength of this approach. Ruetten et al. commented on the usefulness of PED for recurrent HNP based on their experience of 463 cases (19) and subsequently reported its utility (20). Shin et al. (21) conducted revision PED surgery for 41 cases of recurrent HNP after conventional open surgery and reported good to excellent outcomes in over $90 \%$ of cases.

\section{CLINICAL OUTCOME}

The clinical outcome of the present technique was first reported by Yeung and Tsou (9). Of the 307 cases of PED surgery they performed, about $90 \%$ had satisfactory results, which is equivalent to the rate of favorable outcomes in conventional open surgery. Ruetten et al. (22) compared this technique with microdiscectomy and found that the clinical results were comparable in both kinds of surgery, since after endoscopic surgery $82 \%$ of the patients no longer had leg pain and $14 \%$ had only occasional pain.

Birkenmaier et al. (23) reviewed five comparative studies of the present technique and conventional open surgery and concluded that PED had similar clinical outcomes. Moreover, they emphasized the following benefits of PED : shorter operating time, lower estimated blood loss during surgery, fewer surgery-related complications, less surgical site pain immediately after surgery, less need for postoperative pain medication, shorter hospital stay, and a faster return to work.

\section{COMPLICATIONS}

Surgery-related complications are listed in Table 1. Among them, injury to the exiting nerve root is a notable complication of the transforaminal approach, but is very rare when using an interlaminar approach such as Love's technique or MED. This injury occurs in $1.0-8.9 \%$ of cases (24) and there are two possible causes. The first is direct injury by a cannula. When lidocaine infiltrates the exiting nerve root, the patient does not feel any pain, despite injury to the nerve. As a result, dysesthesia and motor paresis will occur just after the surgery. In our initial 100 cases, no patients had this complication as local anesthesia had been carefully performed. The second possible cause is irritation of the dorsal root ganglion due to compression by the cannula during surgery. In this case, dysesthesia of the lower limb develops a couple of days after surgery. In our initial 100 cases, two patients complained of leg pain and dysesthesia 2 days after surgery, although the pain disappeared within 3 months after treatment with medication.

Table 1 : Complications during and after percutaneous endoscopic discectomy with the transforaminal approach.

\begin{tabular}{|c|c|}
\hline \multicolumn{2}{|c|}{ General Complication } \\
\hline Nerve root injury & Cauda equina injury \\
\hline Dural tear & Hematoma \\
\hline Surgical site infection & Major vessel injury \\
\hline \multicolumn{2}{|c|}{ Specific Complication } \\
\hline \multicolumn{2}{|c|}{ Exiting nerve root injury } \\
\hline \multicolumn{2}{|c|}{ Intracranial hypertension } \\
\hline \multicolumn{2}{|c|}{ (Neck pain, headache, convulsion, seizure, death) } \\
\hline \multicolumn{2}{|c|}{ Kidney or colon injury } \\
\hline
\end{tabular}

Another important complication which surgeons must be aware of is intracranial hypertension during surgery, which may result in headache, seizure, or even death. Choi et al. (25) reported 4 cases of seizure among 16,725 cases of PED. The patients complained of neck pain before seizure and therefore neck pain was regarded as an indicator of the consequent seizure. In our initial 100 cases, 2 patients complained of neck pain during surgery. Therefore, we completed surgery as soon as possible and did not experience any further complications such as seizure. Neck pain is an important warning sign of serious complications that can arise from high cranial pressure. 


\section{CASE PRESENTATION}

\section{Case 1}

Figure 7 shows MRI scans before and after surgery in a 32 -year-old male handball player who was not able to play handball due to a herniated mass compressing the L5 nerve root. The mass was removed by PED with the transforaminal approach (Figure 8), and his low back pain disappeared. Within 4 weeks after surgery, stretching and trunk core isometric exercises were started with the assistance of his athletic trainer. From 4 to 8 weeks, mild throwing exercises and jogging were permitted. Eight weeks after the operation, he made a full return to sports activity, including contact sports. To date, he has been active in sports for 6 months after the surgery.

\section{Case 2}

Figure 9 shows MRI scans before and after PED surgery in a 29 -year-old man who had received conventional open surgery for an HNP at the L4/5 level 7 years earlier. When similar symptoms appeared, he visited us and was treated by PED under local anesthesia. The next afternoon, he returned to his sedentary job. The difference in the length of the incisional scar compared with the conventional open procedure is obvious (Figure 10).
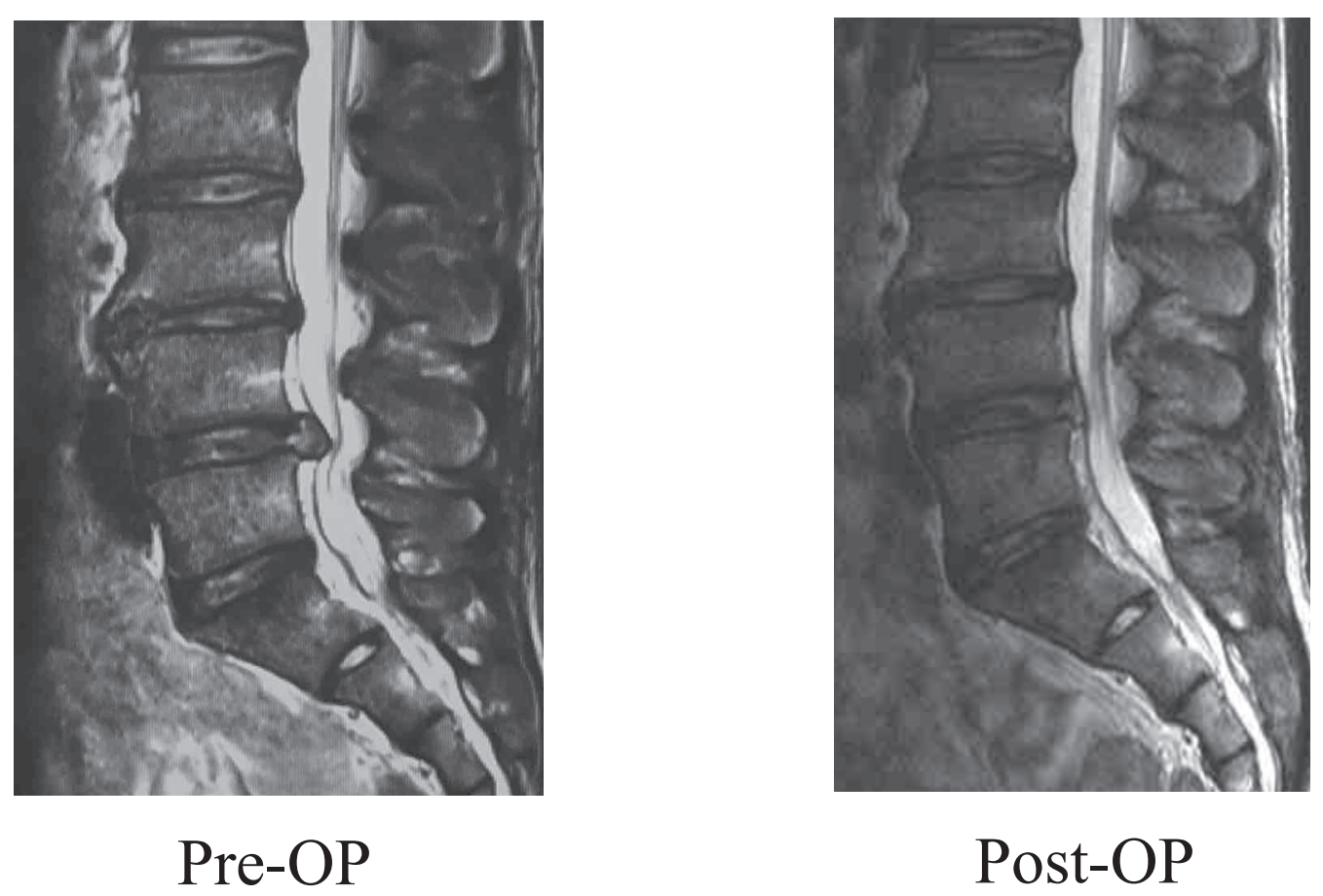

Figure 7 : T2-weighted sagittal view before and after surgery in Case 1.

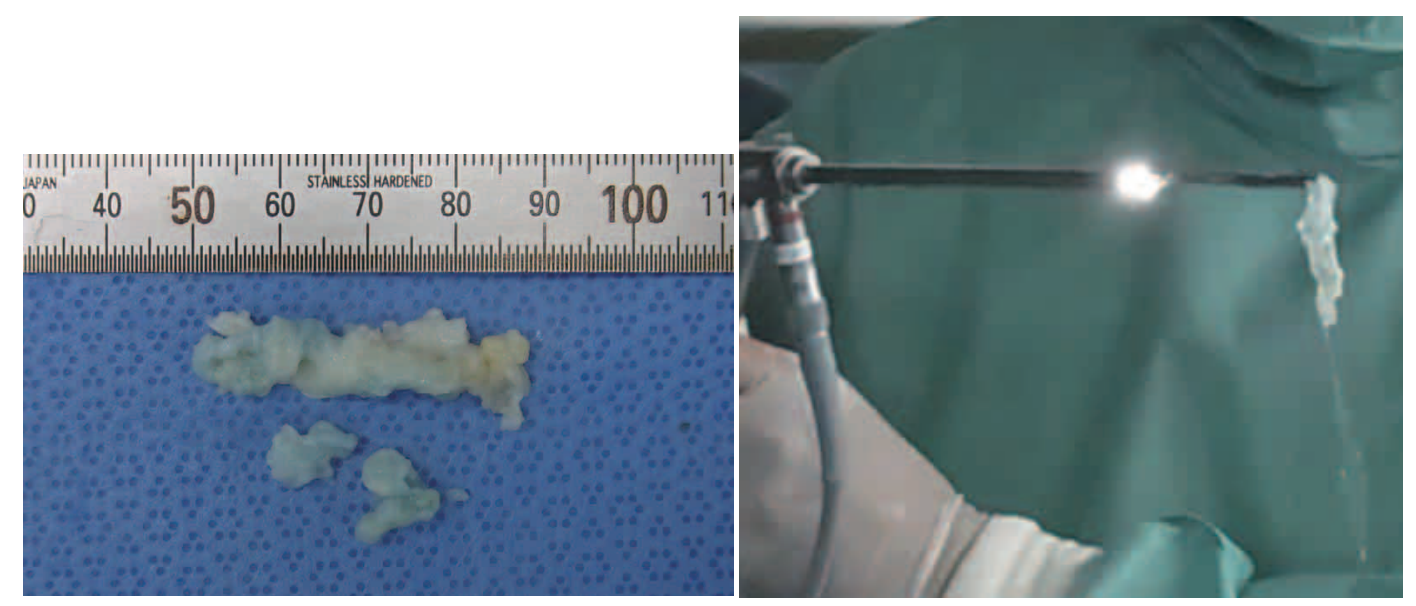

Figure 8 : Herniated fragment removal in Case 1. 


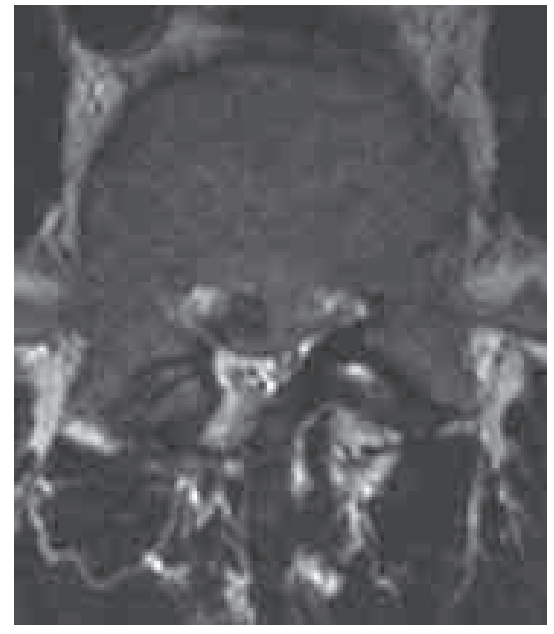

Pre-OP

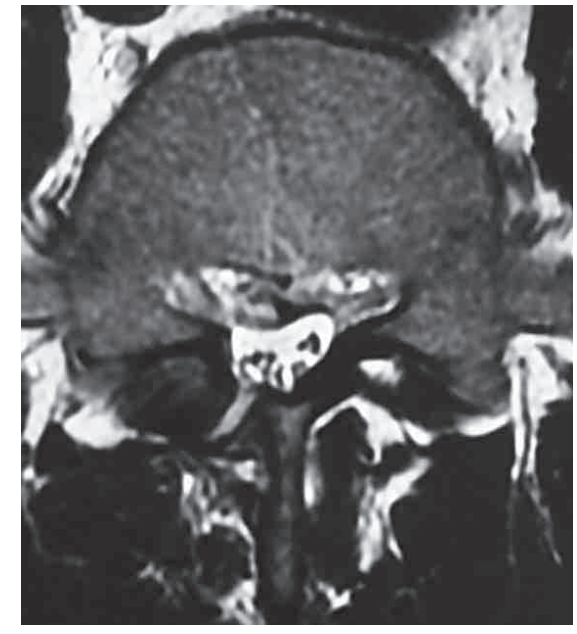

Post-OP

Figure 9 : T2-weighted axial view before and after surgery in Case 2.

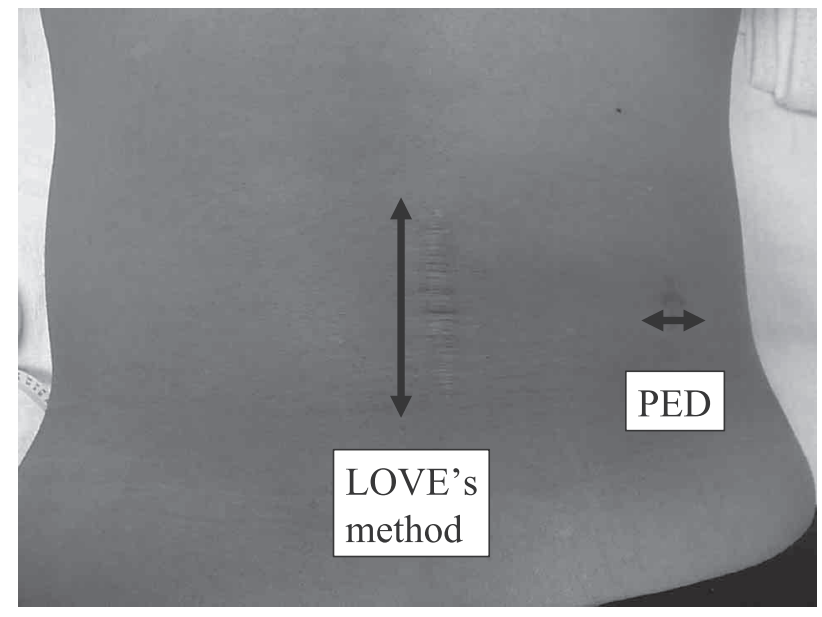

Figure 10 : Skin incisional scar in Case 2 after Love's method and PED.

\section{Case 3}

Figure 11 shows MRI scans before and after PED surgery in a 40-year-old woman who had received micro-Love surgery for an HNP at the L4/5 level 20 years earlier. After carrying a heavy package, she experienced severe leg pain. A recurrent $\mathrm{HNP}$ at the same level was diagnosed for which she underwent PED surgery. The HNP fragment had migrated slightly in the caudal direction. After partial cranial pediculotomy, a cannula was inserted cranially and the migrated mass was removed (Figure 12). The incisional scar of the micro-Love procedure was 3 $\mathrm{cm}$ and that of PED was $8 \mathrm{~mm}$ (Figure 12).
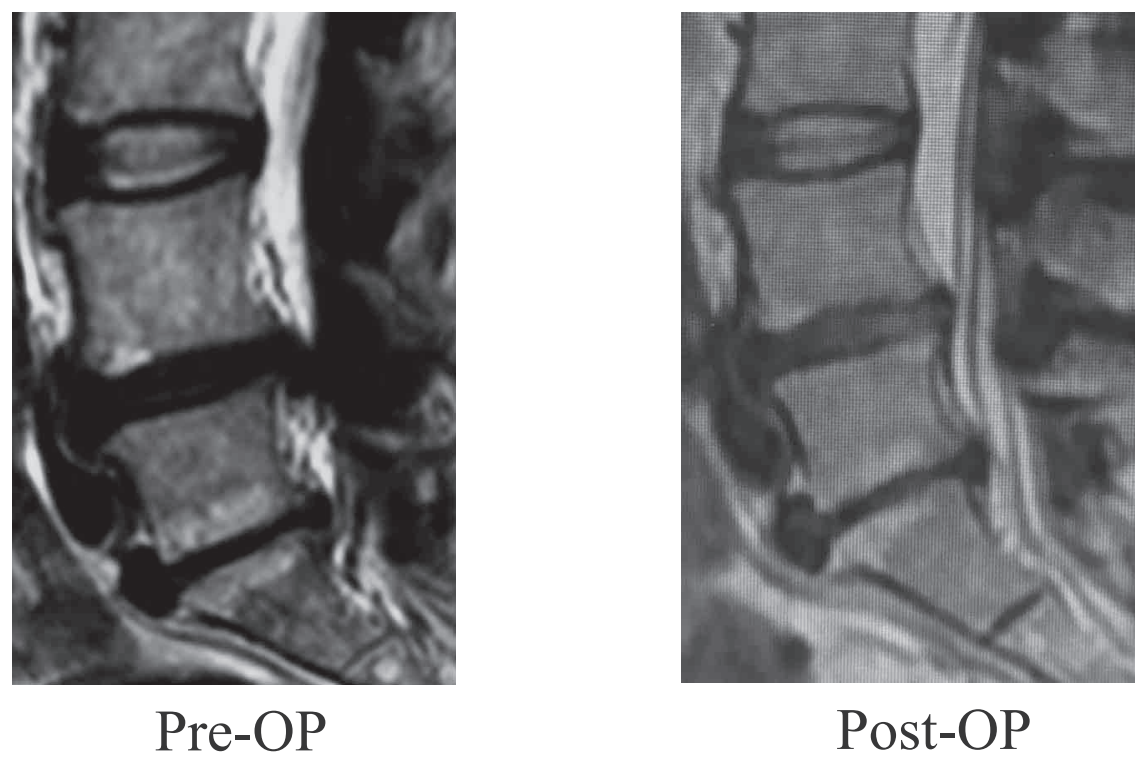

Figure 11 : T2-weighted sagittal view before and after surgery in Case 3. 


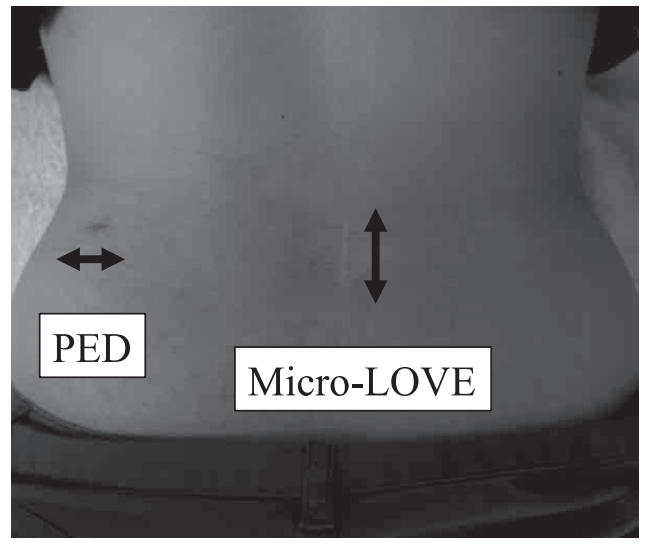

Operation scar

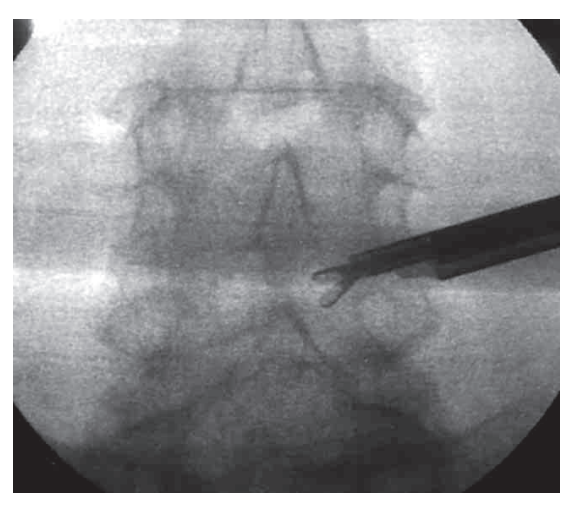

Image intensifier

view

Figure 12 : Skin incisional scar in Case 3 and intraoperative X-ray. Note the difference in surgical scar size between Love's method and PED. The X-ray shows the location of the rongeour after partial pediculectomy.

\section{FUTURE PERSPECTIVES}

Two kinds of new approaches using PED have been introduced. One is laminectomy with a highspeed drill for lumbar spinal canal stenosis (26) and the other is radiofrequency thermal annuloplasty for discogenic low back pain $(14,27)$. However, neither approach has been widely used to date. Therefore, the benefits and details of these new technologies should be disseminated by technical educational seminars using fresh cadaveric spines.

\section{REFERENCES}

1. McCulloch JA : Focus issue on lumbar disc herniation : macro- and microdiscectomy. Spine 21(24 Suppl) : 45S-56S, 1996

2. Foley KT, Smith MM : Microendoscopic discectomy. Tech Neurosurg $3: 301-307,1997$

3. Destandeau J : Technical features of endoscopic surgery for lumbar disc herniation : 191 patients. Neurochirurgie 50(1) : 6-10, 2004

4. Hijikata $\mathrm{S}$ : Percutaneous nucleotomy. A new concept technique and 12 years' experience. Clin Orthop Relat Res 238 : 9-23, 1989

5. Kambin P, Schaffer JL : Percutaneous lumbar discectomy. Review of 100 patients and current practice. Clin Orthop Relat Res $238: 24-34$, 1989

6. Kambin P : Arthroscopic microdiskectomy. Mt Sinai J Med 58(2) : 159-64, 1991

7. Schreiber A, Leu H : Percutaneous nucleotomy : technique with discoscopy. Orthopedics.
1991 Apr ; 14(4) : 439-44

8. Yeung AT : The evolution of percutaneous spinal endoscopy and discectomy : state of the art. Mt Sinai J Med $67: 327-32,2000$

9. Yeung AT, Tsou PM : Posterolateral endoscopic excision for lumbar disc herniation : Surgical technique, outcome, and complications in 307 consecutive cases. Spine 27 : 722-31, 2002

10. Yeung AT, Yeung CA : Minimally invasive techniques for the management of lumbar disc herniation. Orthop Clin North Am 38(3) : 363-72, 2007

11. Dezawa A, Sairyo K : New minimally invasive endoscopic discectomy technique through the interlaminar space using a percutaneous endoscope. Asian J Endosc Surgery 4(2) : 94-8, 2011

12. Dezawa A, Mikami H, Sairyo K : Percutaneous endoscopic translaminar approach for herniated nucleus pulposus in the hidden zone of the lumbar spine. Asian J Endosc Surgery 5(4) : 200-3, 2011

13. Kitahama Y, Sairyo K, Dezawa A : Percutaneous endoscopic transforaminal approach to decompress the lateral recess in an elderly patient with spinal canal stenosis, herniated nucleus pulposus and pulmonary comorbidities. Asian J Endosc Surg 6(2) : 130-3, 2013

14. Sairyo K, Kitagawa Y, Dezawa A : Percutaneous endoscopic discectomy and thermal annuloplasty for professional athletes. Asian J Endosc Surg 6(4) : 292-7, 2013

15. Lee S, Kim SK, Lee SH, Kim WJ, Choi WC, Choi G, Shin SW : Percutaneous endoscopic 
lumbar discectomy for migrated disc herniation : classification of disc migration and surgical approaches. Eur Spine J 16(3) : 431-7, 2007

16. Lee SH, Kang HS, Choi G, Kong BJ, Ahn Y, Kim JS, Lee HY : Foraminoplastic ventral epidural approach for removal of extruded herniated fragment at the L5-S1 level. Neurol Med Chir (Tokyo) 50(12) : 1074-8, 2010

17. Sasani M, Ozer AF, Oktenoglu T, Canbulat N, Sarioglu AC : Percutaneous endoscopic discectomy for far lateral lumbar disc herniations : prospective study and outcome of 66 patients. Minim Invasive Neurosurg 50(2) : 91-7, 2007

18. Kitagawa Y, Sairyo K, Shibuya I, Kitahama Y, Kanamori Y, Koga S, Matsumoto H, Sumita T, Yamada A, Dezawa A : Minimally invasive and simultaneous removal of herniated intracanal and extracanal lumbar nucleus pulposus with a percutaneous spinal endoscope. Asian J Endosc Surg 5(4) : 183-6, 2012

19. Ruetten S, Komp M, Godolias G : An extreme lateral access for the surgery of lumbar disc herniations inside the spinal canal using the full-endoscopic uniportal transforaminal approach-technique and prospective results of 463 patients. Spine $30: 2570-8,2005$

20. Ruetten S, Komp M, Merk H, Godolias G : Recurrent lumbar disc herniation after conventional discectomy : a prospective, randomized study comparing full-endoscopic interlaminar and transforaminal versus microsurgical revision. J Spinal Disord Tech 22(2) : 122-9, 2009

21. Shin KH, Chang HG, Rhee NK, Lim KS : Revisional percutaneous full endoscopic disc surgery for recurrent herniation of previous open lumbar discectomy. Asian Spine J 5(1) : 1-9, 2011

22. Ruetten S, Komp M, Merk H, Godolias G : Full-endoscopic interlaminar and transforaminal lumbar discectomy versus conventional microsurgical technique : a prospective, randomized, controlled study. Spine 33 : 931-9, 2008

23. Birkenmaier C, Komp M, Leu HF, Wegener B, Ruetten S: The current state of endoscopic disc surgery : review of controlled studies comparing full-endoscopic procedures for disc herniations to standard procedures. Pain Physician 16(4) : 335-44, 2013

24. Choi I, Ahn JO, So WS, Lee SJ, Choi IJ, Kim $\mathrm{H}$ : Exiting root injury in transforaminal endoscopic discectomy : preoperative image considerations for safety. Eur Spine J 22(11) : 2481-7, 2013

25. Choi G, Kang HY, Modi HN, Prada N, Nicolau RJ, Joh JY, Pan WJ, Lee SH : Risk of developing seizure after percutaneous endoscopic lumbar discectomy. J Spinal Disord Tech 24(2) : 83-92, 2011

26. Ruetten S, Komp M, Hahn P, Oezdemir S : Decompression of lumbar lateral spinal stenosis : full-endoscopic, interlaminar technique. Oper Orthop Traumatol 25(1) : 31-46, 2013

27. Tsou PM, Alan Yeung C, Yeung AT : Posterolateral transforaminal selective endoscopic discectomy and thermal annuloplasty for chronic lumbar discogenic pain : a minimal access visualized intradiscal surgical procedure. Spine J 4(5) : 564-73, 2004 